\title{
Oncogenic role of microRNA-20a in human uveal melanoma
}

\author{
JINZI ZHOU ${ }^{1}$, JIAN JIANG $^{2}$, SHUHONG WANG $^{1}$ and XIAOBO XIA ${ }^{2}$ \\ ${ }^{1}$ Department of Ophthalmology, Huai'an First People's Hospital, Nanjing Medical University, Huai'an, Jiangsu 223300; \\ ${ }^{2}$ Department of Ophthalmology, Xiangya Hospital Central South University, Changsha, Hunan 410008, P.R. China
}

Received July 8, 2015; Accepted April 25, 2016

DOI: $10.3892 / \mathrm{mmr} .2016 .5433$

\begin{abstract}
As a member of the microRNA (miR)-17-92 cluster, miR-20a has been indicated to be involved in the regulation of the proliferation and invasion of various cancer cells. Previous studies have observed elevated plasma levels of miR-20a in patients with uveal melanoma (UM), compared with normal controls. In the present study, the potential function of miR-20a in UM was investigated. Reverse transcription-quantitative polymerase chain reaction analysis was performed to detect the expression levels of miR-20a in UM cells and tissues. The functions of miR-20a on cell proliferation, migration and invasion were determined in vitro using 3-(4,5-dimethyl-thiazol-2-yl)-2,5-diphenyltetrazolium bromide and Transwell assays, respectively. The expression levels of miR-20a were significantly increased in the UM cells and tissues $(\mathrm{P}<0.05)$. Subsequently, miR-20a mimics were transfected into UM cells, which led to increases in cell growth, migration and invasion activities. By contrast, miR-20a inhibition markedly suppressed the viability and motility of UM cells in vitro. These data provided convincing evidence that miR-20a may function as an oncogenic miRNA, and may be involved in promoting cell growth and motility in the molecular etiology of UM, suggesting its potential as a candidate therapeutic target for the treatment of patients with UM.
\end{abstract}

\section{Introduction}

Uveal melanoma (UM), the most common type of primary intraocular malignancy in adults, is an uncommon tumor with an incidence rate of 5.3-10.9/1,000,000 cases annually (1). It has a high mortality rate of $\sim 50 \%$ due to its high metastatic potential (2). In addition, $\sim 50 \%$ of all patients with UM develop metastatic disease, which is often in the liver (3). In

Correspondence to: Dr Jinzi Zhou, Department of Ophthalmology, Huai'an First People's Hospital, Nanjing Medical University, 6 Beijing Road West, Huai'an, Jiangsu 223300, P.R. China E-mail: jzz_58975@sina.com

Key words: microRNA-20a, uveal melanoma, proliferation, reverse transcription-quantitative polymerase chain reaction assay, migration, invasion clinical practice, the conventional treatments for this disease, including surgical excision, radiotherapy and chemotherapy, have been found to have limited efficacy. For example, early metastases are usually identified 2-5 years following treatment of the primary UM and exhibit poor sensitivity to chemotherapy, subsequently leading to a high mortality rate (4). To improve the clinical outcome of patients with UM, several other treatments, including transpupillary thermotherapy, charged particle irradiation, photocoagulation and immunotherapy, have been developed in the past decade. However, there are no effective therapies for patients with metastasis (5). Thus, it is necessary to investigate the mechanisms underlying the progression of UM, and to identify novel markers for early prognosis and targets for the treatment of this disease.

Increasing evidence has shown that several genetic events, including the abnormal expression of microRNAs (miRNAs), are required for carcinogenesis and cancer progression (6). miRNAs, a group of non-protein-coding RNA molecules of 18-26 nucleotides in length, are endogenously expressed and functionally regulate gene expression post-transcriptionally by directly binding to the specific complementary sequences at target mRNA 3'-untranslated regions (7). Increasing studies have reported that miRNAs are crucial in a variety of biological processes, including cell proliferation, development, differentiation and apoptosis $(8,9)$. In cases of human malignancies, miRNAs act as oncogenes or tumor suppressors. The dysregulation of miRNAs can lead to oncogenesis, with the enhancement of cell proliferation and metastatic potential (10). Accumulating evidence has indicated that miRNAs are associated with the progression of UM. For example, Dong et al (11) reported that miR-34b and miR-34c may have a tumor suppressive role in UM cell proliferation and migration via regulating the expression of multiple targets, including c-Met, phosphorylated-Akt and cell cycle-associated proteins. Liu et al (12) found that miR-9 can suppress UM cell migration and invasion, partly through downregulation of the nuclear factor- $\kappa \mathrm{B} 1$ signaling pathway. Yan et al (13) indicated that miR-182, a p53-dependent miRNA, suppresses the expression of microphthalmia-associated transcription factor, B cell lymphoma 2 (BCL2) and cyclin D (CCND)2, and functions as a potent tumor suppressor in UM cells. These findings suggest that miRNAs may be important in UM via the regulation of cell proliferation, migration, invasion and apoptosis. Further investigations of the involvement of miRNAs in UM may assist in the development of novel and efficient approaches for clinical therapy. 
miR-20a, located on chromosome 13, belongs to the miR-17-92 cluster, members of which have been reported to have oncogenic and tumor suppressor roles (14). The upregulation of miR-20a has been observed in gliomas (15), thyroid cancer (16), gastric cancer (17), colon adenocarcinoma (18), cervical cancer (19) and prostate cancer (20), whereas its downregulation has been found in breast cancer (21), hepatocellular carcinoma (22) and pancreatic carcinoma (23), suggesting its different roles in various human malignancies, with a tissue-specific manner. Functionally, the downregulation of miR-20a can increase the proliferation abilities of hepatocellular carcinoma cells by reducing the endogenous protein level of myeloid cell leukemia sequence 1 (22). The expression level of miR-20a has been found to be higher in more de-differentiated prostate cancer cells, which supports the oncogenic role of miR-20a in prostate cancer carcinogenesis (20). miR-20a can significantly inhibit thyroid cancer cell proliferation in vitro and in vivo, with tumor spheroid formation and invasion in multiple thyroid cancer cell lines (16). miR-20a also promotes the proliferation, migration and invasion of cervical cancer cells through targeting autophagy related 7 and tissue inhibitor of metalloproteinase 2 (19). miR-20a increases the growth, migration and invasion of gastric cancer cells, and enhances the chemoresistance of gastric cancer cells to cisplatin and docetaxel (17). In cases of UM, previous experiments used quantitative polymerase chain reaction (qPCR) assays to compare the plasma and cellular levels of immune regulatory miRNAs between patients with UM and healthy controls, and miR-20a was found to be significantly upregulated in the UM group (24). However, the biological functions of miR-20a in UM remain to be fully elucidated. Thus, the present study evaluated the expression levels of miR-20a in UM tissues and cell lines. Subsequently, the potential roles of miR-20a in UM were determined using gain-of-function and loss-of-function approaches. As a result, the overexpression of miR-20a was observed in the UM cells and tissues, which may have enhanced the cell growth, migration and invasive activities, implying it may have potential as a therapeutic target in this malignancy.

\section{Materials and methods}

Patients and tissue samples. The present study was performed in strict accordance with the recommendations and approval of the Research Ethics Committee of Huai'an First People's Hospital, Nanjing Medical University (Huai'an, China). All patients agreed to the procedure and signed consent forms. All specimens were handled and made anonymous according to the ethical and legal standards.

Informed consent was obtained from all individual participants included in the present study.

Ethical approval. All procedures performed in investigations involving human participants in the present study were in accordance with ethical standards of the institution and/or national research committee, and with the 1964 Helsinki declaration and its later amendments or comparable ethical standards (25).

A total of 10 tumor tissue samples (length, 2.5-10 mm; width, $<12 \mathrm{~mm}$ ) were obtained from 10 patients $(8$ male and 2 female; age range, 36-68 years) with primary UM between
A

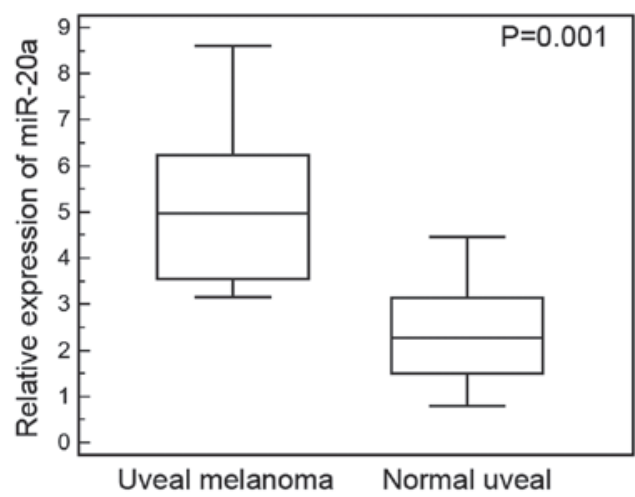

B

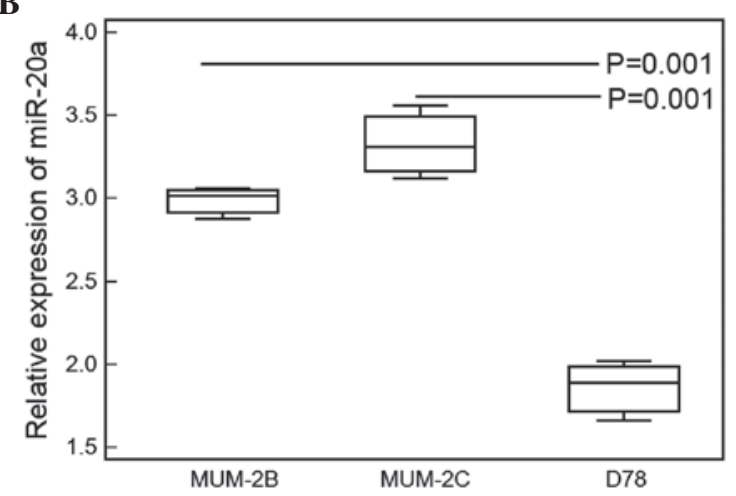

Figure 1. miR-20a is upregulated in human UM tissues and cells. (A) In the human UM tissues, the mean expression level of miR-20a was 5.29 (range, 3.16-8.61). In the normal uveal tissues, the mean expression level of miR-20a was 2.39 (range, 0.68-4.47). Statistical analysis showed that the expression level of miR-20a in the UM tissues was significantly higher, compared with that in normal uveal tissues $(\mathrm{P}=0.001)$. (B) Expression levels of miR-20a were significantly increased in the MUM-2B and MUM-2C UM cell lines, compared with the D78 normal human melanocyte cell line $(\mathrm{P}=0.01)$. Data are presented as the mean \pm standard deviation. UM, uveal melanoma; miR, microRNA.

2010 and 2014 at Huai'an First People's Hospital, Nanjing Medical University, which were immediately frozen in liquid nitrogen. In addition, 10 normal uveal tissue samples (6 male and 4 female; age, 32-70 years) were collected from the Department of pathology in Huai'an First People's Hospital, Nanjing Medical University.

Cell culture and transfection. Two UM cell lines, MUM-2B and MUM-2C, and one normal human melanocyte cell line, D78, were obtained from the Cell Bank of the Chinese Academy of Sciences (Beijing, China). The MUM-2B and MUM-2C cells were originally isolated from the same metastasis of a primary UM. However, the two cell lines exhibit different phenotypes: MUM-2B is epithelioid and highly invasive, whereas MUM-2C is spindle-shaped and shows poor invasiveness. All cells were cultured in Dulbecco's modified Eagle's medium (DMEM; Invitrogen; Thermo Fisher Scientific, Inc., Waltham, MA, USA) supplemented with $10 \%$ fetal bovine serum (FBS; GE Healthcare Life Sciences HyClone Laboratories, Logan, UT, USA) and incubated at $37^{\circ} \mathrm{C}$ in a humidified incubator containing $5 \% \mathrm{CO}_{2}$.

The miR-20a inhibitor and mimics and their controls were synthesized by GenePharma (Shanghai, China) and transfected into cells using Lipofectamine 2000 (Invitrogen; 
A

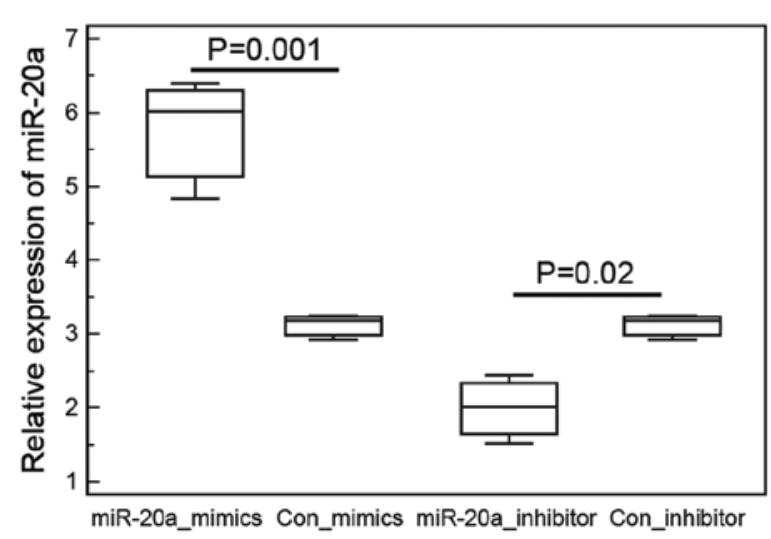

C

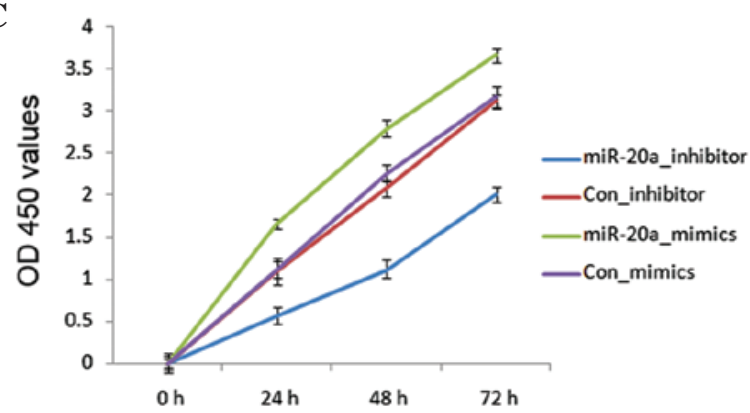

B

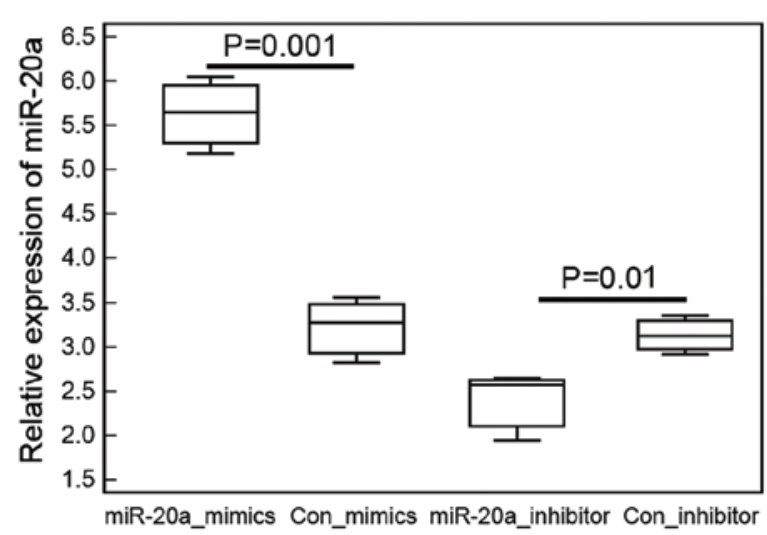

D

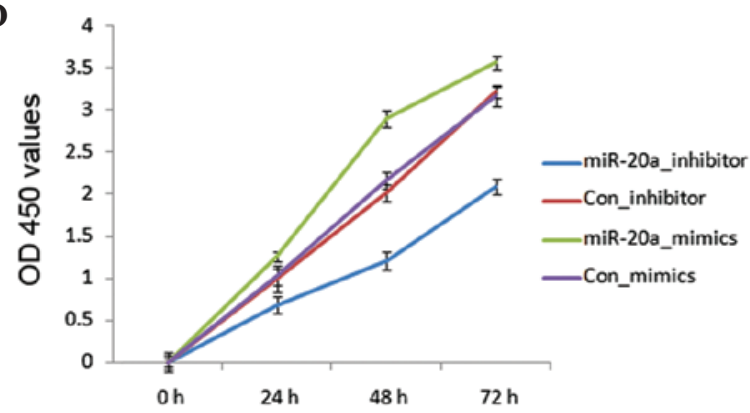

Figure 2. Upregulation of miR-20a promotes UM cell proliferation. The expression levels of miR-20a were significantly elevated in the (A) MUM-2B and (B) MUM-2C cells transfected with miR-20a mimics, but were effectively reduced following transfection with miR-20a inhibitor. In the cell proliferation assay, growth rates were suppressed in the (C) MUM-2B and (D) MUM-2C cells following transfection with miR-20a mimics, with inhibitory efficiencies were 40.38 and $39.66 \%$, respectively. By contrast, the miR-20a inhibitor promoted MUM-2B and MUM-2C cell proliferation. Data are presented as the mean \pm standard deviation. UM, uveal melanoma; miR, microRNA; Con, control; OD, optical density.

Thermo Fisher Scientific, Inc.), according to the manufacturer's protocol.

Reverse transcription-qPCR for the expression of miRNA. Total RNA was isolated from homogenized frozen tissue specimens or the cells using TRIzol reagent (Invitrogen; Thermo Fisher Scientific, Inc.), according to the manufacturer's protocol. The expression levels of miR-20a in the UM tissues and cells were detected using RT-qPCR analysis. The quality of the RNA was evaluated by calculating the RNA integrity number, and $2 \mu \mathrm{g}$ RNA was used for the first-strand cDNA synthesis via reverse transcription. The RT-qPCR was performed using $1 \mathrm{ml}$ cDNA on a Real-Time PCR system (Applied Biosystems Life Technologies, Foster City, CA, USA) in triplicate. U6 was used as an internal control. The sequences of the primers were as follows: miR-20a, forward 5'-TACGATAAAGTGCTTATAGTG CAGGTAG-3' and reverse 5'-GTCCTTGGTGCCCGAGTG-3'; and U6, forward 5'-ATTGGAACGATACAGAGAAGATT-3' and reverse 5'-GTCCTTGGTGCCCGAGTG-3'. Subsequently, the amplification process was performed using a Hairpin-it TM miRNAs qPCR Quantitation kit (GenePharma), according to the manufacturer's protocol. The $20 \mu \mathrm{l}$ reaction mixture used for qPCR contained 12.5 $\mu \mathrm{l}$ SYBR Green supermix, $3.5 \mu \mathrm{l}$ RNase-free water, $1 \mu 1$ forward primers, $1 \mu 1$ reverse primers and $2 \mu \mathrm{l}$ of the reverse transcribed product. The reactive conditions were as follows: 40 amplification cycles of $95^{\circ} \mathrm{C}$ for $3 \mathrm{~min}$, $95^{\circ} \mathrm{C}$ for $12 \mathrm{sec}$ and $62^{\circ} \mathrm{C}$ for $50 \mathrm{sec}$.
The relative expression levels of miR-20a were qualified using the comparative quantification cycle (Cq) method (23). Each sample was examined in triplicate and the normalized gene expression, presented as the mean \pm standard deviation, was calculated from three independent experiments.

Cell proliferationassay. A3-(4,5-dimethyl-thiazol-2-yl)-2,5-diphenyltetrazolium bromide assay (Sigma-Aldrich, St. Louis, MO, USA) was used to evaluate the proliferation activities of the UM cells with or without transfection with miR-20a inhibitor, mimics and their controls. Each sample was examined in triplicate. The normalized gene expression, presented as the mean \pm standard deviation, was calculated from three independent experiments.

Cell invasion assay. A cell invasion assay was performed to evaluate the invasive activities of the UM cells with or without transfection with miR-20a inhibitor, mimics and their controls. In brief, the Transwell plates (EMD Millipore, Billerica, MA, USA) were incubated to solidify with Matrigel (BD Biosciences, San Jose, CA, USA) at $37^{\circ} \mathrm{C}$ for $6 \mathrm{~h}$. A total of $1 \times 10^{5}$ cells were suspended in serum-free DMEM and, after $24 \mathrm{~h}$ at room temperature, were added into the upper chamber. Subsequently, medium containing $10 \%$ FBS was added to the lower chamber. After $24 \mathrm{~h}$ at room temperature, the invasive cells on the lower chamber were stained with hematoxylin and eosin and counted. Images of the invaded cells were captured using a photomicroscope (Olympus Corporation, 
A

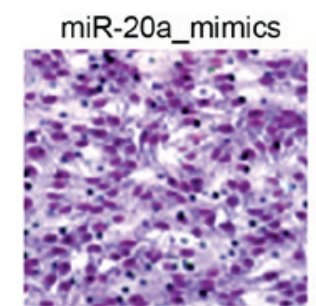

miR-20a_inhibitor

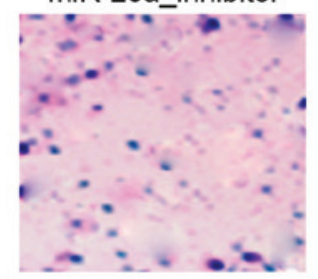

B

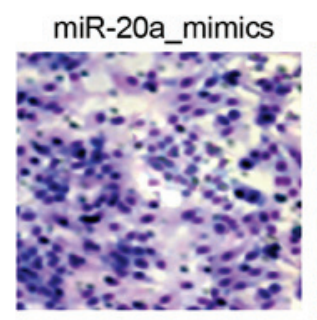

miR-20a_inhibitor

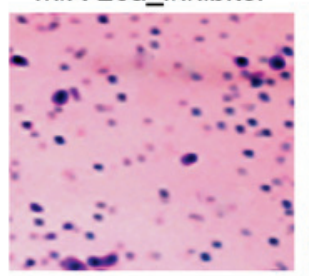

Con_mimics

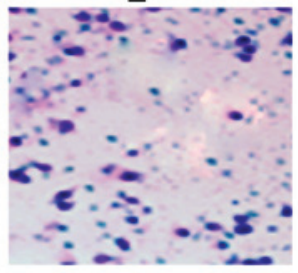

Con_inhibitor

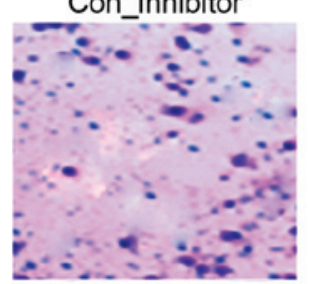

Con_mimics

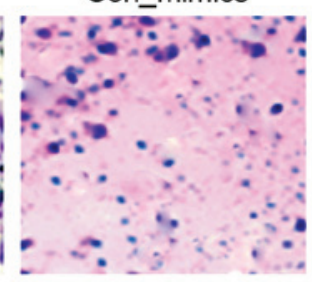

Con_inhibitor

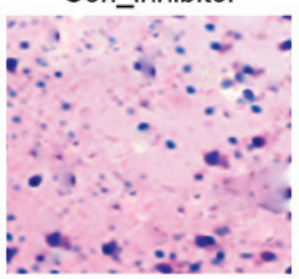

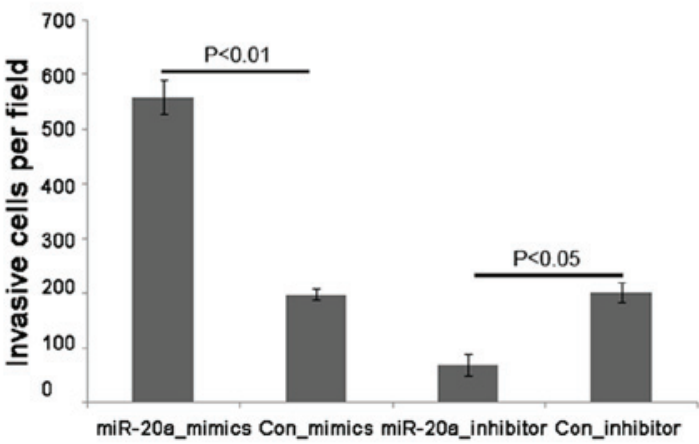

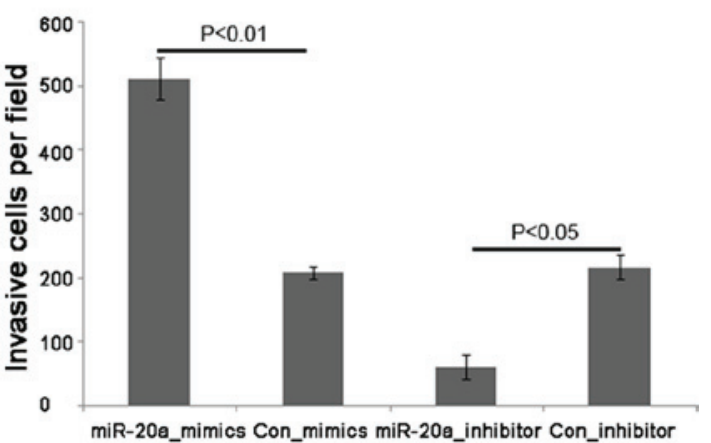

Figure 3. Upregulation of miR-20a promotes UM cell invasion. The invasiveness of the (A) MUM-2B and (B) MUM-2C cells were increased following transfection with the miR-20a mimics, compared with the control mimics, but were significantly decreased by transfection with the miR-20a inhibitor, compared with the control group $(\mathrm{P}<0.05)$. Cells were stained with hematoxylin and eosin (magnification, $\mathrm{x} 100)$. Data are presented as the mean \pm standard deviation. UM, uveal melanoma; miR, microRNA; Con, control.

Tokyo, Japan). Each sample was examined in triplicate. The normalized gene expression, presented as the mean \pm standard deviation, was calculated from three independent experiments

Cell migration assay. A cell migration assay was performed to evaluate the migration activities of the UM cells with or without transfection with miR-20a inhibitor, mimics and their controls. In brief, the cells $\left(1 \times 10^{5}\right.$ cells $/ 100 \mu 1$ serum-free medium) were added to the upper chamber of $8 \mu \mathrm{m}$ pore size Transwell plates (EMD Millipore) following the transfection. DMEM containing $10 \%$ FBS was added to the lower chamber as a chemoattractant. After $24 \mathrm{~h}$ at room temperature, the migrated cells attached to the bottom were stained with hematoxylin and eosin and counted. Images of the invaded cells were captured using a photomicroscope (Olympus Corporation). Each sample was examined in triplicate. The normalized gene expression, presented as the mean \pm standard deviation, was calculated from three independent experiments.

Databases. The present study used the miRTarBase (release 4.5: Nov. 1, 2013; http://mirtarbase.mbc.nctu.edu.tw/), which has accumulated $>50,000$ miRNA-target interactions (MTIs) (26), to collect validated targets for miR-20a. The present study performed pathway enrichment analysis based on the Kyoto Encyclopedia of Genes and Genomes (KEGG) database (http://www.genome.jp/kegg/; last updated: Oct 16, 2012) (27).

Statistical analysis. All statistical analyses were performed using SPSS version 16.0 software for Windows (SPSS, Inc., Chicago, IL, USA). Data are expressed as the mean \pm standard deviation. Differences between two groups were determined using Student's $t$-test and differences in more than two groups were determined using one-way analysis of variance. $\mathrm{P}<0.05$ was considered to indicate a statistically significant difference.

\section{Results}

miR-20a is upregulated in human UM tissues and cells. In the human UM tissue samples, the mean expression level of miR-20a was 5.29 (range, 3.16-8.61). For the normal uveal tissues, the mean expression level of miR-20a was 2.39 (range, $0.68-4.47)$. Statistical analysis showed that the expression level of miR-20a in the UM tissues was significantly higher, compared with that in the normal uveal tissues $(\mathrm{P}=0.001$; Fig. 1A). Similarly, the expression of miR-20a was also mark- 
A
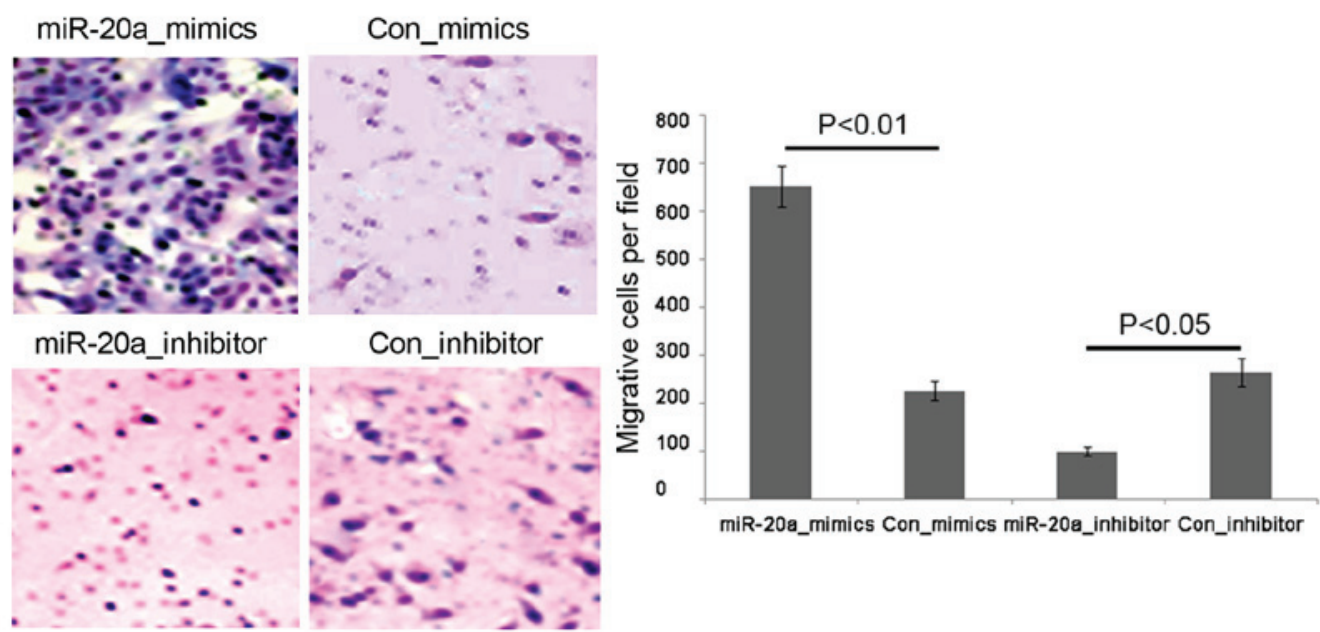

B
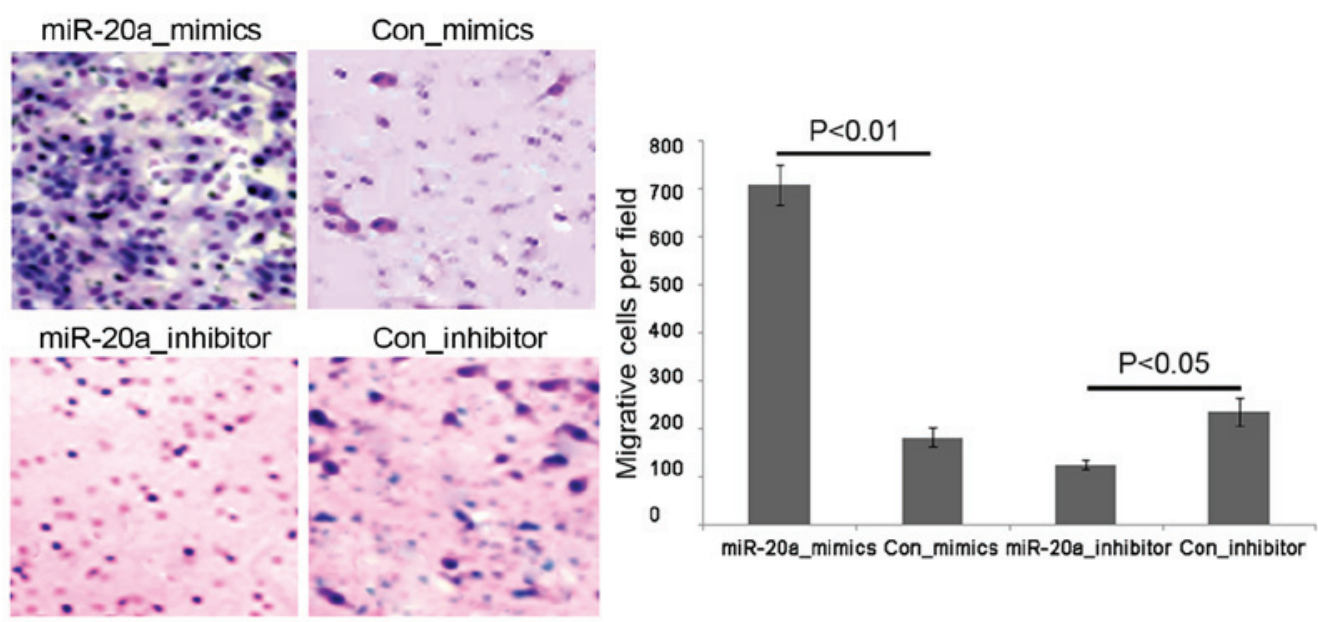

Figure 4. Upregulation of miR-20a promotes UM cell migration. The migration abilities of the (A) MUM-2B and (B) MUM-2C cells were increased following tranfection with the miR-20a mimics, compared with the control mimics, but were significantly decreased by transfection with the miR-20a inhibitor, compared with the control group $(\mathrm{P}<0.05)$. Cells were stained with hematoxylin and eosin (magnification, $\mathrm{x} 100)$.Data are presented as the mean \pm standard deviation. UM, uveal melanoma; miR, microRNA; Con, control.

edly increased in the UM cell lines (MUM-2B and MUM-2C), compared with the normal human melanocyte D78 cell line ( $\mathrm{P}=0.01$; Fig. 1B).

Upregulation of miR-20a promotes UM cell proliferation. To investigate whether the upregulation of miR-20a is important in UM cell proliferation, the MUM-2B and MUM-2C cells were transfected with miR-20a mimics or miR-20a inhibitor. As shown in Fig. 2A and B, the expression levels of miR-20a were significantly elevated in the MUM-2B and MUM-2C cells transfected with miR-20a mimics, but was effectively reduced following transfection with the miR-20a inhibitor. In the cell proliferation assay, the growth rate was increased in the MUM-2B and MUM-2C cells following transfection with miR-20a mimics, with promotion efficiencies of 40.38 and $39.66 \%$, respectively (Fig. 2C and D). By contrast, the miR-20a inhibitor suppressed MUM-2B and MUM-2C cell proliferation (Fig. 2C and D).

Upregulation of miR-20a promotes UM cell motility. The invasiveness of MUM-2B and MUM-2C cells were increased following transfection with miR-20a mimics, compared with the control mimics, but were markedly decreased by the miR-20a inhibitor, compared with the control group (Fig. 3A and B for MUM-2B and MUM-2C cells, respectively; $\mathrm{P}<0.05)$.

Similarly, miR-20a-induced migration was significantly increased in the miR-20a mimic-transfected cells, compared with the mimic-control-transfected cells (Fig. 4A and B) for MUM-2B and MUM-2C cells, respectively; $\mathrm{P}<0.05$ ). By contrast, when miR-20a inhibitor was transfected into the MUM-2B and MUM-2C cells, the miR-20a inhibitor effectively inhibited the miR-20a-induced promotion of the migration abilities of the UM cells (Fig. 4A and B for MUM-2B and MUM-2C cells, respectively; $\mathrm{P}<0.05$ ).

\section{Discussion}

The development of UM is a multistep process involving genetic and epigenetic alterations of proto-oncogenes and tumor suppressor genes. Previous studies have increased understanding of the implications of miRNAs in this type of malignancy, and found that miRNAs can control the malignant phenotypes of UM cells, including cell-cycle progression, apoptosis, invasiveness and metastasis (28-30). In addition, emerging evidence has shown a correlation between the 
expression of several miRNAs, and the initiation and progression of UM. Thus, it is of important clinical significance to elucidate the biological aspects of miRNA dysregulation to improve current understanding of the pathogenesis of UM and develop miRNA-directed therapeutics against this malignancy. In the present study, the data showed the upregulation of miR-20a in human UM cells and tissues. Specifically, the ectopic expression of miR-20a promoted UM cell proliferation and motility in vitro. In addition, the miR-20a inhibitor effectively suppressed UM cell proliferation and motility in vitro. These findings suggested that miR-20a had an oncogenic role in promoting the progression of UM.

miR-17-92, one of the most well-characterized oncogenic miRNA clusters, contains six mature miRNAs: miR-17, miR-18a, miR-19a, miR-20a, miR-19b-1 and miR-92-1, which are integrated components of several cancer-associated signaling pathways (31-33). Among them, the upregulation of miR-20a has been detected in multiple human cancer tissues, malignant cell culture systems and animal models $(34,35)$. Previous reports have also revealed the ability of miR-20a to regulate several cellular processes and to promote malignant transformation $(36,37)$. In the present study, it was observed that miR-20a was frequently overexpressed in the UM tissues and cell lines, which prompted the hypothesis that miR-20a may be a novel tumor oncogenic miRNA, and its dysregulation may be involved in the aggressive progression of human UM. To validate this, the present study investigated the function of miR-20a in two UM cell lines, MUM-2B and MUM-2C, following the construction of stable miR-20a-mimics and miR-20a inhibitor UM cell lines. As expected, the enforced and inhibited expression of miR-20a promoted and prevented tumor progression, respectively, by modulating cell proliferation, invasion and migration.

Due to the fact that miRNAs exert their biological functions via regulating the expression of their targets, the present study collected the validated targets of miR-20a from miRTarBase. Generally, the collected MTIs are validated experimentally using a reporter assay, western blot analysis, qPCR, microarrays and next-generation sequencing experiments. In the present study, only the MTIs, which were validated experimentally using reporter assays, western blot analysis and qPCR, were collected. As a result, 29 genes, including amyloid precursor protein, Rho GTPase activating protein $12, \mathrm{BCL} 2$, bone morphogenetic protein receptor 2 , BCL2/adenovirus E1B 19kDa interacting protein 2, CCND1, CCND2, cyclin-dependent kinase inhibitor 1A, E2F1, E2F3, Egl-9 family hypoxia-inducible factor 3, hypoxia-inducible factor $1 \alpha$, mitogen-activated protein kinase (MAPK) kinase kinase 12, MAPK9, myocyte enhancer factor 2D, MYC, phosphatase and tensin homolog, purine-rich element binding protein $\mathrm{A}$, retinoblastoma(RB)1, RB-like (RBL)1, RBL2, Runt-related transcription factor 1, signal-regulatory protein $\alpha$, transforming growth factor $\beta$ receptor 2 , tumor susceptibility 101, vascular endothelial growth factor A and WEE1, have been experimentally validated as candidate targets for miR-20a. As a single miRNA can reduce the expression of multiple targets, which often belong to the same signaling pathway, the present study performed pathway enrichment analysis based on the KEGG database. These candidate targets for miR-20a were significantly associated with cell cycle ( $\mathrm{P}=2.06 \mathrm{E}-08)$, the phosphoinositide 3 -kinase-Akt signaling pathway $(\mathrm{P}=5.25 \mathrm{E}-05)$ and focal adhesion $(\mathrm{P}=4.58 \mathrm{E}-05)$, which have all been reported to be involved in the tumor progression and metastatic cascade of UM.

Taken together, the data of the present study provided convincing evidence that miR-20a may function as an oncogenic miRNA and were involved in promoting cell growth and motility in the molecular etiology of UM, suggesting its potential as a candidate therapeutic target for patients with UM. However, there were two limitations; the sample size of the clinical cohort was small. To overcome this limitation, further investigations and additional samples are required to confirm the clinical significance of miR-20a in UM. Secondly, the exact downstream target genes of miR-20a in UM cells was not validated, and further investigation to elucidate the molecular mechanisms underlying the action of miRNA on UM is warranted.

\section{References}

1. Field MG and Harbour JW: Recent developments in prognostic and predictive testing in uveal melanoma. Curr Opin Ophthalmol 25: 234-239, 2014.

2. Schoenfield L: Uveal melanoma: A pathologist's perspective and review of translational developments. Adv Anat Pathol 21: 138-143, 2014.

3. Eschelman DJ, Gonsalves CF and Sato T: Transhepatic therapies for metastatic uveal melanoma. Semin Intervent Radiol 30: 39-48, 2013.

4. Rashid $\mathrm{AB}$ and Grossniklaus HE: Clinical, pathologic, and imaging features and biological markers of uveal melanoma. Methods Mol Biol 1102: 397-425, 2014.

5. Werdich XQ, Jakobiec FA, Singh AD and Kim IK: A review of advanced genetic testing for clinical prognostication in uveal melanoma. Semin Ophthalmol 28: 361-371, 2013.

6. Yan X, Xu H and Yan Z: Functional perspective and implications of gene expression by noncoding RNAs. Cancer Transl Med 1: 137-152, 2015.

7. Chen Y, Zhao H, Tan Z, Zhang C and Fu X: Bottleneck limitations for microRNA-based therapeutics from bench to the bedside. Pharmazie 70: 147-154, 2015.

8. Mansoori B, Mohammadi A, Shirjang S and Baradaran B: Micro-RNAs: The new potential biomarkers in cancer diagnosis, prognosis and cancer therapy. Cell Mol Biol (Noisy-le-grand). 61: 1-10, 2015.

9. Hata A and Lieberman J: Dysregulation of microRNA biogenesis and gene silencing in cancer. Sci Signal 8: re3, 2015.

10. Shen J and Hung MC: Signaling-mediated regulation of MicroRNA processing. Cancer Res 75: 783-791, 2015.

11. Dong F and Lou D: MicroRNA-34b/c suppresses uveal melanoma cell proliferation and migration through multiple targets. Mol Vis 18: 537-546, 2012.

12. Liu N, Sun Q, Chen J, Li J, Zeng Y, Zhai S, Li P, Wang B and Wang X: MicroRNA-9 suppresses uveal melanoma cell migration and invasion through the NF- $\kappa \mathrm{B} 1$ pathway. Oncol Rep 28: 961-968, 2012.

13. Yan D, Dong XD, Chen X, Yao S, Wang L, Wang J, Wang C, $\mathrm{Hu}$ DN, Qu J and Tu L: Role of microRNA-182 in posterior uveal melanoma: Regulation of tumor development through MITF, BCL2 and cyclin D2. PLoS One 7: e40967, 2012.

14. Ventura A, Young AG, Winslow MM, Lintault L, Meissner A, Erkeland SJ, Newman J, Bronson RT, Crowley D, Stone JR, et al: Targeted deletion reveals essential and overlapping functions of the miR-17 through 92 family of miRNA clusters. Cell 132: 875-886, 2008.

15. Wang Z, Wang B, Shi Y, Xu C, Xiao HL, Ma LN, Xu SL, Yang L, Wang QL, Dang WQ, et al: Oncogenic miR-20a and miR-106a enhance the invasiveness of human glioma stem cells by directly targeting TIMP-2. Oncogene 34: 1407-1419, 2015.

16. Xiong Y, Zhang L and Kebebew E: MiR-20a is upregulated in anaplastic thyroid cancer and targets LIMK1. PLoS One 9: e96103, 2014 
17. Li X, Zhang Z, Yu M, Li L, Du G, Xiao W and Yang H: Involvement of miR-20a in promoting gastric cancer progression by targeting early growth response 2 (EGR2). Int J Mol Sci 14: 16226-16239, 2013.

18. Schetter AJ, Leung SY, Sohn JJ, Zanetti KA, Bowman ED, Yanaihara N, Yuen ST, Chan TL, Kwong DL, Au GK, et al: MicroRNA expression profiles associated with prognosis and therapeutic outcome in colon adenocarcinoma. JAMA 299: 425-436, 2008

19. Zhao S, Yao D, Chen J, Ding N and Ren F: MiR-20a promotes cervical cancer proliferation and metastasis in vitro and in vivo. PLoS One 10: e0120905, 2015.

20. Qiang XF, Zhang ZW, Liu Q, Sun N, Pan LL, Shen J, Li T, Yun C, $\mathrm{Li} \mathrm{H}$ and Shi LH: miR-20a promotes prostate cancer invasion and migration through targeting ABL2. J Cell Biochem 115: $1269-1276,2014$.

21. Yu Z, Wang C, Wang M, Li Z, Casimiro MC, Liu M, Wu K, Whittle J, Ju X, Hyslop T, et al: A cyclin D1/microRNA 17/20 regulatory feedback loop in control of breast cancer cell proliferation. J Cell Biol 182: 509-517, 2008.

22. Zhang Y, Zheng L, Ding Y, Li Q, Wang R, Liu T, Sun Q, Yang H, Peng S, Wang W and Chen L: MiR-20a induces cell radioresistance by activating the PTEN/PI3K/Akt signaling pathway in hepatocellular carcinoma. Int J Radiat Oncol Biol Phys 92: $1132-1140,2015$

23. Hong TH and Park IY: MicroRNA expression profiling of diagnostic needle aspirates from surgical pancreatic cancer specimens. Ann Surg Treat Res 87: 290-297, 2014

24. Achberger S, Aldrich W, Tubbs R, Crabb JW, Singh AD and Triozzi PL: Circulating immune cell and microRNA in patients with uveal melanoma developing metastatic disease. Mol Immunol 58: 182-186, 2014.

25. Puri KS, Suresh KR, Gogtay NJ and Thatte UM: Declaration of Helsinki, 2008: Implications for stakeholders in research. J Postgrad Med. 55: 131-134, 2009.

26. Hsu SD, Tseng YT, Shrestha S, Lin YL, Khaleel A, Chou CH, Chu CF, Huang HY, Lin CM, Ho SY, et al: miRTarBase update 2014: An information resource for experimentally validated miRNA-target interactions. Nucleic Acids Res 42 (Database Issue): D78-D85, 2014.
27. Wixon $\mathrm{J}$ and Kell D: The Kyoto encyclopedia of genes and genomes-KEGG. Yeast 17: 48-55, 2000.

28. Qureshi R and Sacan A: A novel method for the normalization of microRNA RT-PCR data. BMC Med Genomics 6 (Suppl 1): S14, 2013

29. Li Z, Yu X, Shen J and Jiang Y: MicroRNA dysregulation in uveal melanoma: A new player enters the game. Oncotarget 6: 4562-4568, 2015.

30. Eldh M, Olofsson Bagge R, Lässer C, Svanvik J, Sjöstrand M, Mattsson J, Lindnér P, Choi DS, Gho YS and Lötvall J: MicroRNA in exosomes isolated directly from the liver circulation in patients with metastatic uveal melanoma. BMC Cancer 14: 962, 2014

31. Grillari J, Hackl M and Grillari-Voglauer R: miR-17-92 cluster: Ups and downs in cancer and aging. Biogerontology 11: 501-506, 2010.

32. Xie R, Lin X, Du T, Xu K, Shen H, Wei F, Hao W, Lin T, Lin $\mathrm{X}$, Qin Y, et al: Targeted disruption of miR-17-92 impairs mouse spermatogenesis by activating mTOR signaling pathway. Medicine (Baltimore) 95: e2713, 2016

33. Li L, Shi JY, Zhu GQ and Shi B: MiR-17-92 cluster regulates cell proliferation and collagen synthesis by targeting TGFB pathway in mouse palatal mesenchymal cells. J Cell Biochem 113: 1235-1244, 2012.

34. Zhang GJ, Li Y, Zhou H, Xiao HX and Zhou T: miR-20a is an independent prognostic factor in colorectal cancer and is involved in cell metastasis. Mol Med Rep 10: 283-291, 2014.

35. Zhuo W, Ge W, Meng G, Jia S, Zhou X and Liu J: MicroRNA-20a promotes the proliferation and cell cycle of human osteosarcoma cells by suppressing early growth response 2 expression. Mol Med Rep 12: 4989-4994, 2015.

36. Wei W, Hou J, Alder O, Ye X, Lee S, Cullum R, Chu A, Zhao Y, Warner SM, Knight DA, et al: Genome-wide microRNA and messenger RNA profiling in rodent liver development implicates mir302b and mir20a in repressing transforming growth factor-beta signaling. Hepatology 57: 2491-2501, 2013.

37. Ristau J, Staffa J, Schrotz-King P, Gigic B, Makar KW, Hoffmeister M, Brenner H, Ulrich A, Schneider M, Ulrich CM and Habermann N: Suitability of circulating miRNAs as potential prognostic markers in colorectal cancer. Cancer Epidemiol Biomarkers Prev 23: 2632-2637, 2014 